\title{
A THEORY OF COTYPES'
}

\author{
BY ARTHUR SARD \\ Communicated by Richard Palais, March 11, 1969
}

1. Introduction. Suppose that $M$ is a manifold, modeled on a Banach space $X$. For each subset $S$ of $M$, we will define a cotype, which will be a nonnegative integer or $\infty$. The larger cotype $S$, the smaller $S$. The theory of cotypes will permit us to distinguish sizes of large subsets of $M$.

Cotype is a generalization of Baire category. Just as Baire's theory is based on sets whose closure contains no sphere, the theory of cotypes is based on sets whose closure contains no diffeomorph of a coball in $X$ of specified codimension. We use the words diffeomorphism and diffeomorph in the following sense: $\phi$ is a $C^{q}$-diffeomorphism of $M$ into a manifold $N$ (modeled on a Banach space $Y$ ) iff $\phi$ is a bijective map, $C^{a}$ in both directions, whose domain is open in $M$ and whose range is open in $N$. If $\phi$ exists, then $X$ and $Y$ must be isomorphic. If a special term is desired, $\phi$ may be called a full diffeomorphism.

As an application, we cite a theorem on the cotype of the image of the critical set of a Fredholm map of negative index. The theorem is suggested by and is close to one of Smale's.

The definition of cotype will be relative to a differentiability class $C^{q}$. The reader may wish to take $q=1$ throughout. It is quite possible that cotype is independent of $q$, for $q \geqq 1$. That is, if $M$ is a $C^{q}$-manifold, $q \geqq 1$, modeled on $X$, and if $S \subset M$, then cotype $S$ may be unchanged if we increase $q$ by changing the atlas of $M$. This is likely to be the case, if $X$ is finite dimensional [4]. Our theory is directed, however, towards infinite dimensional manifolds. Cotype for $q=0$ may be different from cotype for $q=1$.

2. Cotype. Let $M$ be a $C^{a}$-manifold, with countable basis, modeled on a Banach space $X$ of more than one point, with or without boundary.

By a set $\boldsymbol{\gamma}_{r} \subset M$, we shall always mean a diffeomorph of an $r$-coball, $r=0,1, \cdots$. That is,

$$
\gamma_{r}=\phi \Gamma_{r},
$$

where $\phi$ is a $C^{a}$-diffeomorphism of $X$ into $M$, and $\Gamma_{r}$ is an $r$-coball,

\footnotetext{
${ }^{1}$ Research supported in part by the United States Atomic Energy Commission.
} 
that is, the diametral section of a ball in $X$ with center 0 , by a linear subspace of codimension $r$. The domain of $\phi$ is required to be an open set of $X$ containing $\Gamma_{r}$ and the range of $\phi$ an open set of $M$.

Let $S$ be an arbitrary subset of $M$. We shall define cotype $S$. To do this, we first define the relations

$$
\cot S \geqq r \quad \text { and } \quad \text { cotype } S \geqq r, \quad r=0,1, \cdots,
$$

as follows.

We say that $\cot S \geqq 0$ always; and that $\cot S \geqq r+1$ if the closure $\bar{S}$ of $S$ does not contain a set $\gamma_{r}$. Thus cot $S \geqq 1$ iff $S$ is rare. And $\cot S \geqq r+1$ implies that $\cot S \geqq r$. If $T \subset S$ and $\cot S \geqq r$, then $\cot T \geqq r$.

Next we say that cotype $S \geqq r$ if $S$ is the countable union of sets each of which is of cot $\geqq r$. The adjective countable shall always mean finite or countably infinite. As cot $0 \geqq p$ for all integers $p$, it follows that cotype $S \geqq r$ iff $S_{1}, S_{2}, \cdots$ exist such that

$$
S=S_{1} \cup S_{2} \cup \cdots, \quad \cot S_{j} \geqq r, \quad j=1,2, \cdots .
$$

If cotype $S \geqq r+1$, then cotype $S \geqq r$. If $T \subset S$ and cotype $S \geqq r$, then cotype $T \geqq r$. If $\cot S \geqq r$, then cotype $S \geqq r$.

Finally, we say that cotype $S=r$ if cotype $S \geqq r$ and if it is not true that cotype $S \geqq r+1$. We say that cotype $S=\infty$ if cotype $S \geqq r$ for all $r$. Thus

$$
\text { cotype } S=\sup \{r: \operatorname{cotype} S \geqq r\} \text {. }
$$

Thus, cotype $S=r, 1 \leqq r<\infty$, iff $S$ is the countable union of sets whose separate closures contain no set $\gamma_{r-1}$ and, furthermore, whenever $S$ is written as a countable union of sets, at least one of the latter has a closure which contains a set $\gamma_{r}$. And cotype $S=0$ iff $S$ is not meager. And cotype $S=\infty$ iff, for each $r \geqq 1, S$ may be written as the countable union, possibly depending on $r$, of sets whose separate closures contain no set $\gamma_{r-1}$.

Also,

$$
\begin{aligned}
& \text { cotype } 0=\infty, \\
& \text { cotype }\{\text { solo point }\}=\operatorname{dimension} M, \\
& \text { cotype } M=0 .
\end{aligned}
$$

A countable union of sets of cotype $\geqq r$ is itself of cotype $\geqq r$; and if one of the sets is of cotype $r$, then so is the union. Hence a set of cotype $r<\infty$ is not the countable union of sets of cotype $\geqq r+1$.

Cotype $S$, as now defined, is greater than or equal to $r$ iff cotype $S \geqq r$, as earlier defined. 
THEOREM. Cotype is invariant under diffeomorphism.

Proof. Let $\phi$ be a full diffeomorphism of $M$ into a $C^{q}$-manifold $N$, with countable basis, modeled on a Banach space. The domain $D$ of $\phi$ is open in $M$; the range, open in $N$. We shall show that if $S \subset D$, then cotype $S=$ cotype $\phi S$.

For this it is sufficient to show that, for $r=0,1, \cdots$, cotype $\phi S \geqq r$ if cotype $S \geqq r$. Suppose that cotype $S \geqq r$. Then $S=\cup_{\mu=1,2}, \ldots S_{\mu}$ and $\cot S_{\mu} \geqq r$. Now $S_{\mu} \subset D$, but $\bar{S}_{\mu}$ need not be. As $M$ has a countable basis, we may by Lindelöf's theorem subdivide $S_{\mu}$ into countably many subsets $S_{\mu, \lambda} \lambda=1,2, \cdots$, such that $\bar{S}_{\mu, \lambda} \subset$ D. Then

$$
S=\underset{\mu, \lambda}{\bigcup} S_{\mu, \lambda}, \quad \cot S_{\mu, \lambda} \geqq r,
$$

since $S_{\mu, \lambda} \subset S_{\mu}$. And $\phi \bar{S}_{\mu, \lambda}=\bar{\phi}_{\mu, \lambda}$. Hence cot $\phi S_{\mu, \lambda} \geqq r$, else $\phi \bar{S}_{\mu, \lambda}$ and therefore $\bar{S}_{\mu, \lambda}$ would contain a set $\gamma_{r-1}$. Since $\phi S=\bigcup \phi S_{\mu, \lambda}$, it follows that cotype $\phi S \geqq r$.

For the rest of the paper we assume that $q \geqq 1$.

Lemma. Cotype $\gamma_{r}=r, r=0,1, \cdots ; r \leqq \operatorname{dim} M$.

Proof. By the invariance of cotype under diffeomorphism, it is sufficient to show that cotype $\Gamma_{r}=r$, where $\Gamma_{r}$ is an $r$-coball in $X$.

Now cot $\Gamma_{r} \geqq r$. This is certainly true for $r=0$. Take $r>0$. Assume, as will be absurd, that $\bar{\Gamma}_{r} \supset \gamma_{r-1}$. Then by shrinkage $\Gamma_{r}$ contains (a different) $\gamma_{r-1}$. Let $\Gamma_{r-1}$ be an $(r-1)$-coball which contains $\Gamma_{r}$. Then $\Gamma_{r}$ and hence $\gamma_{r-1}$ are meager in $\Gamma_{r-1}$. On the other hand, $\gamma_{r-1}=\theta \Gamma_{r-1}$, where $\theta$ is a diffeomorphism of $X$ to $X$. And $\theta$ restricted to $\Gamma_{r-1}$ is a diffeomorphism on $\Gamma_{r-1}$ into $\Gamma_{r-1}$, because the inverse function theorem assures that $\theta$ as restricted is an open map. Hence $\gamma_{r-1}$ contains an interior point in $\Gamma_{r-1}$ and cannot be meager in $\Gamma_{r-1}$. Hence $\cot \Gamma_{r} \geqq r$.

Hence cotype $\Gamma_{r} \geqq r$. Finally, suppose that

$$
\Gamma_{r}=\underset{\mu=1,2, \ldots}{\bigcup} S_{\mu}
$$

We shall show that for some $\mu, \bar{S}_{\mu} \supset \gamma_{r}$. Now $\Gamma_{r}=B \cap R_{r}$, where $B$ is a ball in $X$ and $R_{r}$ is a subspace of $X$ of codimension $r$. Baire's theorem for the space $R_{r}$ implies that for some $\mu, \bar{S}_{\mu}$ contains a ball in $R_{r}$, which is an $r$-coball in $X$. Hence it is impossible that cotype $\Gamma_{r} \geqq r+1$. Hence cotype $\Gamma_{r}=r$.

Corollary. Sets $\gamma_{r}$ and $\gamma_{s}$ in $M, r, s \leqq \operatorname{dim} M$, are diffeomorphic iff $r=s$. 
This completes our construction of the theory of cotypes. There is a dual theory of types, in which we say that $\operatorname{tip} S \leqq p, p=0,1, \cdots$, $S \subset M$, if $\bar{S}$ contains no diffeomorph of a $(p+1)$-ball and type $S \leqq p$ if $S$ is the countable union of sets each of tip $\leqq p$. Just as the theory of cotypes distinguishes large sets in $M$, the theory of types distinguishes small sets. But the theories of Hausdorff measure and topological dimension also distinguish small sets and may perhaps do so more usefully than the theory of types [8].

Frank Quinn has constructed a theory of conullity which, like the theory of cotypes, distinguishes large subsets of manifolds [personal communication].

3. Fredholm maps. Suppose that $M$ and $N$ are $C^{q}$-manifolds, $q \geqq 1$, with or without boundaries, with countable bases, modeled on Banach spaces $X$ and $Y$, respectively. Let $f: M \rightarrow N$ be a $C^{q_{-}}$-map of $M$ into $N$. We say that $x \in M$ is a critical point of $f$ if the tangent map $D f(x): X \rightarrow Y$ is neither injective nor onjective (surjective). Let $A$ denote the set of critical points of $f$.

Kupka [3] has shown that the critical image $f A$ need not be small compared to the entire image $f M$, if $M$ is infinite dimensional, even if $q=\infty$; cf. [1] also. Thus an additional hypothesis on $f$ is needed to assure a conclusion that $f A$ is small. One such hypothesis, introduced by Smale, is that $f$ be a Fredholm map [2], [5]. As the index of a Fredholm map is locally constant, the index will be constant on any connected component of the domain. Thus a condition that the index of a Fredholm map be constant is not burdensome: a Fredholm map can be decomposed into maps for each of which the index is constant.

SMALE's TheORem [10]. If $f$ is a Fredholm map of constant index $i$ and if $q \geqq i+1$, then $f A$ is meager in $N$. And $f M$ is nonmeager, if $M \neq A$ and $i \geqq 0$; whereas $f M$ is meager if $i<0$.

The theory of cotypes permits one to establish the following theorem, useful in the case of negative index.

THEOREM. If $f$ is a Fredholm map of constant negative index $i=-j \leqq 0$, then cotype $f A \geqq j+1$. And cotype $f M=j$ if $M \neq A$.

My original proof (unpublished) of this theorem [9] was based on a theorem of 1942 that the image of the points of rank 0 of a $C^{q}$-map of an open set of $R^{m}$ into $R^{n}$ is $m / q$-null [7], and on the following lemma.

Lemma. Let $R_{\delta}$ be a linear subspace of codimension $\delta$ of a Banach space $Y$. Let $\gamma_{j}$ be a $C^{q}$-diffeomorph in $Y$ of a $j$-coball in $Y, j \leqq \delta$. Con- 
sider all cross sections of $\boldsymbol{\gamma}_{j}$ in a direction complementary to $R_{\delta}$. At least one such cross section contains a $C^{q}$-diffeomorph of $a(\delta-j)$-ball.

The proof of this lemma depends on the following consequence of the rank theorem. If $g$ is a $C^{q}$-map of a nonempty open set of $R^{m}$ into $R^{n}, m \geqq n$, then for at least one $c \in R^{n}$, the inverse image $g^{-1}(c)$ contains a $C^{q}$-diffeomorph in $R^{m}$ of an $(m-n)$-ball.

A second proof of the theorem, due to a referee, deduces it from Smale's theorem for the case of index 0 , by local projections. The method of projections, however, reveals less of the fine structure of the critical image than does the direct method of the original proof. I am grateful to the referee for pointing out that the theory of cotypes applies to Banach manifolds rather than merely to Hilbert manifolds as in my original paper.

A third proof of the theorem deduces it from an unpublished theorem of F. Quinn's, in which the map is allowed to be sigmaproper, left Fredholm [6], and the critical image is shown to be of suitable conullity.

\section{BIBLIOGRAPHY}

1. R. Bonic, $A$ note on Sard's theorem in Banach spaces, Proc. Amer. Math. Soc. 17 (1966), 1218.

2. I. C. Gohberg and M. G. Krein, The basic propositions on defect numbers, root numbers, and indices of linear operators, Uspehi Mat. Nauk 12 (1957), no. 2 (74), 43$118=$ Amer. Math. Soc. Transl. (2) 13 (1960), 185-264.

3. I. Kupka, Counter example to the Morse-Sard theorem in the case of infinitedimensional manifolds, Proc. Amer. Math. Soc. 16 (1965), 954-957.

4. J. R. Munkres, Elementary differential topology, Princeton Univ. Press, Princeton, N. J., 1963.

5. R. S. Palais, Seminar on the Atiyah-Singer index theorem, Princeton Univ. Press, Princeton, N. J., 1965.

6. F. Quinn, "Transversal approximation on Banach manifolds," in Global analysis, Proc. Sympos. Pure Math., vol. 15, Amer. Math. Soc., Providence, R. I. (to appear).

7. A. Sard, The measure of the critical values of differentiable maps, Bull. Amer. Soc. 48 (1942), 883-890.

8. - Hausdorff measure of critical images on Banach manifolds, Amer. J. Math 87 (1965), 158-174, 760.

9. - Critical images of maps of negative index, with a theory of cotypes, Abstract, Notices Amer. Math. Soc. 16 (1969), 94.

10. S. Smale, An infinite dimensional version of Sard's theorem, Amer. J. Math. 87 (1965), 861-866.

Queens College, City University of New York, Flushing, New York 11367 